\title{
Active control of multiscale features in wall-bounded turbulence
}

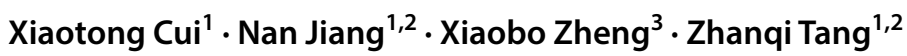

Received: 26 March 2019 / Revised: 23 June 2019 / Accepted: 17 September 2019 / Published online: 5 November 2019 (c) The Author(s) 2019

\begin{abstract}
This study experimentally investigates the impact of a single piezoelectric (PZT) actuator on a turbulent boundary layer from a statistical viewpoint. The working conditions of the actuator include a range of frequencies and amplitudes. The streamwise velocity signals in the turbulent boundary layer flow are measured downstream of the actuator using a hot-wire anemometer. The mean velocity profiles and other basic parameters are reported. Spectra results obtained by discrete wavelet decomposition indicate that the PZT vibration primarily influences the near-wall region. The turbulent intensities at different scales suggest that the actuator redistributes the near-wall turbulent energy. The skewness and flatness distributions show that the actuator effectively alters the sweep events and reduces intermittency at smaller scales. Moreover, under the impact of the PZT actuator, the symmetry of vibration scales' velocity signals is promoted and the structural composition appears in an orderly manner. Probability distribution function results indicate that perturbation causes the fluctuations in vibration scales and smaller scales with high intensity and low intermittency. Based on the flatness factor, the bursting process is also detected. The vibrations reduce the relative intensities of the burst events, indicating that the streamwise vortices in the buffer layer experience direct interference due to the PZT control.
\end{abstract}

Keywords Turbulent boundary layer $\cdot$ Piezoelectric actuator $\cdot$ Drag reduction $\cdot$ Discrete wavelet decomposition $\cdot$ Bursting process

\section{Introduction}

Turbulence control offers significant advantages in various engineering applications. One of the most important techniques used to improve the efficiency of a working system is to reduce the surface drag caused by turbulent boundary layer (TBL) flow, and understanding TBL flow mechanisms is beneficial for the development of control methods. Quasistreamwise vortices (QSVs) are typical coherent structureinducing [1-3] bursting events that dominate the near-wall region, and are associated with most surface friction production [4-6]. The ejection process occurs on the updraft side of QSVs, wherein the low-speed fluids in the inner layer

Nan Jiang

nanj@tju.edu.cn

1 Department of Mechanics, Tianjin University, Tianjin 300354, China

2 Tianjin Key Laboratory of Modern Engineering Mechanics, Tianjin 300354, China

3 School of Naval Architecture Ocean \& Civil Engineering, Shanghai Jiao Tong University, Shanghai 200240, China are lifted away from the wall to the outer layer with the transfer of the mass, energy, momentum, and vortices [7]. The sweep process occurs on the downdraft side of QSVs, wherein the high-speed fluids move downward to the wall, which is directly related to an increase in the skin friction $[8,9]$. Thus, QSVs are the main factors that increase the surface friction, so altering the QSVs is a potential approach for achieving drag reduction $[10,11]$.

Numerous passive and active control methods have been developed based on this flow mechanism [12]. As a representative of the passive control methods, Wash and Choi et al. [13-15] achieved considerable drag reduction on riblet surfaces by conducting experiments and direct numerical simulations. In comparison with the passive control method, the active control method has wider adaptability to complex flows, and it enhances the control effectiveness [16-19], which has been confirmed by several experimental and simulation investigations [20-29]. Bai et al. [2] used a spanwisealigned array of piezoceramic actuators to generate a transverse traveling wave along the wall surface. The actuator can induce a layer of highly regularized streamwise vortices to break the connection between the large-scale coherent 
structures and the wall, achieving a maximum drag reduction of 50\%. Berger et al. [30] obtained a drag reduction of $40 \%$ via an open loop-controlled oscillating spanwise Lorentz force that disturbed the semi-equilibrium state between the near-wall streamwise vortices and the wall in channel flow. Zheng et al. [19] applied a single PZT actuator to break the near-wall streamwise vortices and achieved a drag reduction of $27 \%$. Herein, we employed an active strategy based on the aforementioned achievements.

A basic property of turbulence is its multiscale characteristics. However, few studies have investigated the influence of active control on the TBL's multiscale features. We provided a periodic perturbation to the TBL via a PZT actuator. To further observe the modification in the TBL's multiscale characteristics because of the PZT actuator, we conducted hot-wire measurements. This study reports the drag reduction results with a PZT actuator over a wide range of frequencies and amplitudes, and the multiscale properties of the turbulence are analyzed. Finally, the control effect on the bursting process is observed.

\section{Experimental setup and parameters}

Figure 1 shows the experimental setup. The experiments were conducted in a closed loop wind tunnel. TBL flow developed along a flat plate, which was mounted vertically in the test section. Pitch angle was finely adjusted to ensure a zero-pressure gradient in the streamwise direction. A tripwire was fixed at $x=80 \mathrm{~mm}$ downstream of the leading edge, and a sandpaper band was attached following the tripwire until $x=530 \mathrm{~mm}$. The freestream velocity was $U_{\infty}=9 \mathrm{~m} / \mathrm{s}$. TBL was fully developed at the streamwise location of $x=1000 \mathrm{~mm}$, wherein the end of the control mechanism and measurement was conducted at $x=1002 \mathrm{~mm}$, as shown in Fig. 1. The thickness of the TBL was approximately $\delta=39.8 \mathrm{~mm}$. Based on $U_{\infty}$ and momentum thickness, $\theta$, Reynolds number, $R e_{\theta}$, was 2183.

The PZT actuator was firmly pasted over the plate. The upper surface of the actuator was leveled with the plate's surface. The effective length, thickness, and width of the actuator was $30,0.42$, and $3.62 \mathrm{~mm}$, respectively. The actuator comprised a $220-\mu \mathrm{m}$-thick PZT-5H material and $200-\mu \mathrm{m}$-thick phosphor-copper shim. The basic geometric information and material properties of the PZT actuator are listed in Table 1. The PZT oscillator was excited via a power source, which worked as a cantilever beam. The frequency and amplitude of the actuating voltage were adjustable over a wide range.

We tested three different experimental conditions, wherein the frequencies of actuation were set to 80,160 , and $240 \mathrm{~Hz}$ under the same voltage of $100 \mathrm{~V}$. The corresponding amplitudes of the actuator were $0.4,0.6$, and $0.2 \mathrm{~mm}$, respectively, which were captured using a high-speed camera. The measuring equipment comprised an IFA-300 with a TSI-1621A-T1.5 miniature boundary layer probe $[31,32]$. The sensing element

Table 1 Details of the PZT oscillator materials

\begin{tabular}{lc}
\hline Quantity & Value \\
\hline PZT density $\rho_{\mathrm{p}}\left(\mathrm{kg} \cdot \mathrm{m}^{-3}\right)$ & $7.45 \times 10^{3}$ \\
Shim density $\rho^{\mathrm{s}}\left(\mathrm{kg} \cdot \mathrm{m}^{-3}\right)$ & $8.89 \times 10^{3}$ \\
PZT elastic modulus $E_{\mathrm{p}}\left(\mathrm{N} \cdot \mathrm{m}^{-2}\right)$ & $7.69 \times 10^{10}$ \\
Shim elastic modulus $E_{\mathrm{s}}\left(\mathrm{N} \cdot \mathrm{m}^{-2}\right)$ & $11.3 \times 10^{10}$ \\
PZT strain constant $d_{31}\left(\mathrm{~m} \cdot \mathrm{V}^{-1}\right)$ & $-186 \times 10^{-12}$ \\
\hline
\end{tabular}

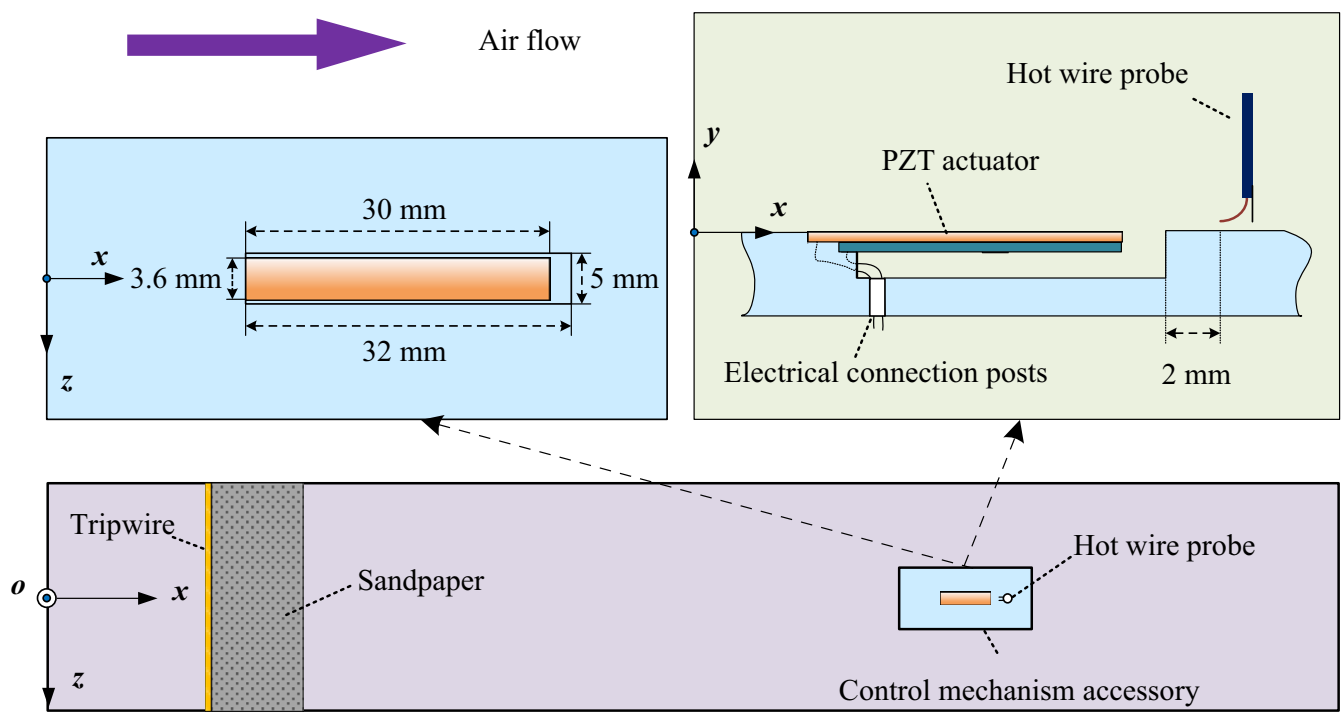

Fig. 1 Schematic of the experimental setup 
of the probe was a tungsten wire with a diameter of $4 \mu \mathrm{m}$ and length $1.25 \mathrm{~mm}$. The calibration was conducted using an air velocity calibrator over the velocity range $0-15 \mathrm{~m} / \mathrm{s}$. Sampling rate and low pass cut-off frequency were 100 and $50 \mathrm{kHz}$, respectively. We obtained time sequences of the streamwise velocity signal at 74 different wall-normal locations. Each sequence comprised $2^{22}$ velocity samples or approximately $42 \mathrm{~s}$ [33].

\section{Results and discussion}

\subsection{Mean velocity profile}

The outer scaled mean velocity profile, scaled using $\delta$ and $U_{\infty}$, is linear in the viscous sublayer [34], as shown in Fig. 2a. The profile slope is related to the skin-friction stress $\tau_{w}$, according to

$\tau_{w}=\rho v \partial U / \partial y$,

where the air flow density is $1.205 \mathrm{~kg} / \mathrm{m}^{3}$ and kinematic viscosity coefficient $v$ is $1.5 \times 10^{-5} \mathrm{~m}^{2} / \mathrm{s}$ [35-37]. The friction velocity, $u_{\tau}$, can be obtained as follows

$u_{\tau}=\sqrt{\bar{\tau}_{w} / \rho}$,

where the overbar denotes time averaging. Figure $2 b$ shows the mean velocity profiles normalized by $u_{\tau}$, and $U^{+}=y^{+}$ is evident for both uncontrolled and controlled cases over $y^{+}=3-5$. The local skin-friction reduction is defined as follows

$\Delta_{\tau_{w}}=\frac{\left(\bar{\tau}_{w}\right)_{\mathrm{on}}-\left(\bar{\tau}_{w}\right)_{\mathrm{off}}}{\left(\bar{\tau}_{w}\right)_{\mathrm{off}}} \times 100 \%$,

where the subscripts on and off denote measurements with and without control, respectively. Table 2 lists the drag

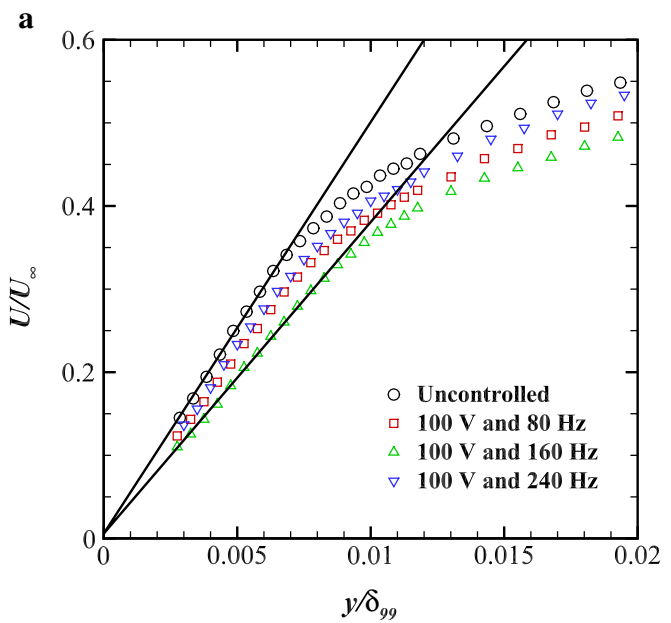

reduction results for different cases. It can be seen that case 2 with the PZT frequency of $160 \mathrm{~Hz}$ and amplitude of $0.6 \mathrm{~mm}$ achieves the maximum drag reduction of $26.4 \%$.

\subsection{Wavelet energy distribution}

The effects of the PZT actuator on multiscale characteristics of the turbulence were investigated by wavelet analysis, which is one of the most widely used multiscale signal processing methods. The definition of this method fits well with the physical characteristics of turbulence [38, 39]. Wavelet analysis includes a continuous wavelet transform (CWT) and discrete wavelet transform (DWT). To improve the computing efficiency and avoid redundant information, DWT was employed for data processing [40].

The wavelet transforms of a signal $u(t)$ with scale $r$ at position $t$ are defined as follows [41]

$w(r, t)=C_{\Psi}^{-1 / 2} r^{-1 / 2} \int_{\infty}^{\infty} \Psi^{*}\left(\frac{s-t}{r}\right) u(s) \mathrm{d} s$,

where $\Psi(t)$ is wavelet function, which satisfies the following admissibility condition

$C_{\Psi}=\int_{\infty}^{\infty}|k|^{-1}|\hat{\Psi}(k)|^{2}<\infty$,

Table 2 Local skin drag reduction

\begin{tabular}{llllll}
\hline Case & $U_{\infty}(\mathrm{m} / \mathrm{s})$ & $f(\mathrm{~Hz})$ & $A(\mathrm{~mm})$ & $u_{\tau}(\mathrm{m} / \mathrm{s})$ & $\Delta_{\tau_{w}}$ \\
\hline Uncontrolled & 9 & 0 & 0 & 0.4317 & 0 \\
1 & 9 & 80 & 0.4 & 0.3971 & $-15.4 \%$ \\
2 & 9 & 160 & 0.6 & 0.3693 & $-26.8 \%$ \\
3 & 9 & 240 & 0.2 & 0.4210 & $-4.9 \%$ \\
\hline
\end{tabular}

b

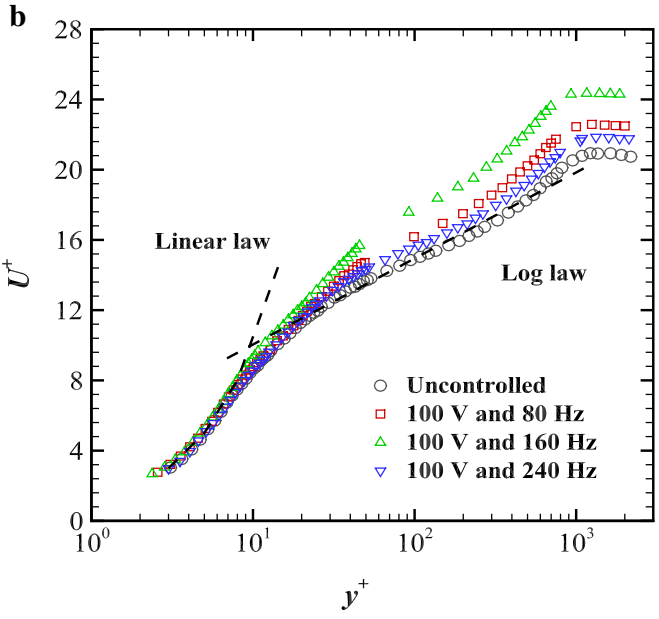

Fig. 2 Distributions of the mean streamwise velocity in the $\mathbf{a}$ outer and $\mathbf{b}$ inner scaled units 
where $\hat{\Psi}(k)$ denotes the Fourier transform of $\Psi(t)$. Equation (5) is the invertibility condition, which is a necessary condition for the signal $u(t)$ to be reconstructed from its wavelet coefficients.

Instead of using CWT, the function $u(t)$ can be decomposed into a series of self-similar orthonormal functions obtained via dilatations and translations of a mother function $\Psi_{00}$ [42]. By choosing separations $r_{k}=2^{-k}$, a set of functions forming the orthonormal basis can be written as follows

$\Psi_{k, j}(t)=-2^{-k / 2} \Psi_{00}\left(2^{k} t-j\right)$.

If $u(t)$ is sampled on a discrete mesh $t_{j}$, the transformed function can be rewritten in a discrete form as follows

$u(j)=\sum_{r=1}^{\infty} \sum_{i=-\infty}^{\infty} w^{r}(i) \Psi^{(r)}\left(i-2^{r} j\right)$,

where $r$ represents the discretized scale. The wavelet function $\Psi^{(r)}\left(i-2^{r} j\right)$ should obey the following orthogonality condition:

$$
\sum_{k=-\infty}^{\infty} \Psi^{(r)}\left(k-2^{r} i\right) \Psi^{(p)}\left(k-2^{p} j\right)=\delta_{i j} \delta_{r p}
$$

Then the coefficients $w^{(r)}(i)$ can be obtained as

$w^{(r)}(i)=\sum_{j=-\infty}^{\infty} \Psi^{r}\left(i-2^{r} j\right) u(j)$.

The reconstructed signal at every scale can be computed as follows

$u(j)^{r}=\sum_{i=-\infty}^{\infty} w^{r}(i) \Psi^{(r)}\left(i-2^{r} j\right)$

In this experiment, DWT in the fluctuating velocity signals was calculated using Daubechies 4 wavelet function. Figure 3 shows the linear relation between the logarithmic characteristic frequency $f_{r}^{w}$ and scale level $r$.

Figure 4 shows the percentage of the reconstructed fluctuations' energy with respect to the total energy, $\left\langle u_{r}^{\prime 2}\right\rangle /\left\langle u^{\prime 2}\right\rangle$, which is a function of the scale levels and wall-normal positions. In Fig. $4 \mathrm{a}$, the uncontrolled case indicates that the inner peak appears at $y^{+}=10-14$ at a scale level of 9 , corresponding to the most energetic structures in the near-wall turbulence, i.e., the QSVs. Figure $4 \mathrm{~b}-\mathrm{d}$ show the results of the controlled cases. It can be seen that the PZT vibration concentrates the energy at scale levels of 10,9 , and 8 , corresponding to the vibration frequencies of 80,160 , and $240 \mathrm{~Hz}$, respectively, which

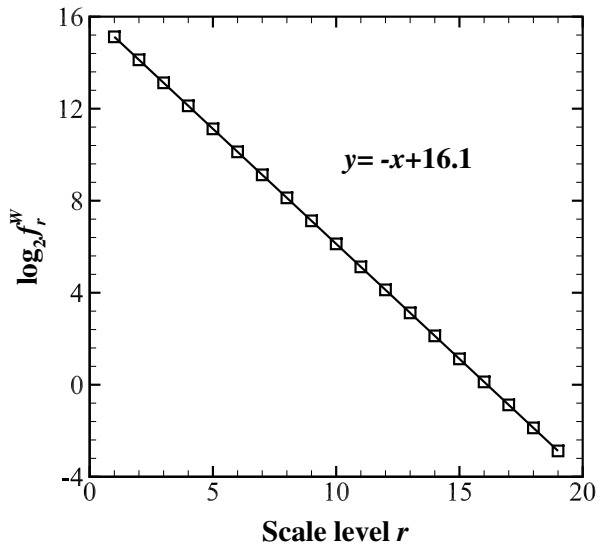

Fig. 3 Relation between the scale level $r$ and characteristic frequency $f_{r}^{w}$

means that the PZT actuator, as expected, has a periodic impact on the downstream flow fields where the hot-wire is measured. As shown, the wall-normal region perturbed by the PZT actuator primarily exists at $y^{+}<40$.

According to Wallace et al. [26], typical bursting events, i.e., sweeps and ejections, are closely correlated with each other at a wall-normal position of 15 wall units. In our study, the vibrations affected this position range. The turbulent energy distribution, as shown in Fig. 4, indicates that the impact of the actuator was concentrated in the range $y^{+}=10-15$. Therefore, the time series of the original and reconstructed fluctuating signals at $y^{+}=14$ in the uncontrolled case and case $2(100 \mathrm{~V}$ and $160 \mathrm{~Hz})$ were compared to investigate the effects of PZT on the scale corresponding to the vibration frequency. In Fig. 5a, b, it can be seen that the original fluctuation $u^{+}$overlaps better with the reconstructed fluctuation $u_{r}^{+}$at a scale level of 9, i.e., corresponding to $160 \mathrm{~Hz}$, in the controlled case than that in the uncontrolled case. This implies that the fluctuation components at this dominating scale are coupling to the PZT actuation.

\subsection{Turbulent intensities}

First, some definitions need to be stated for clarity. The vibration scales indicate the scales approximately corresponding to the vibration frequencies 80,160 , and $240 \mathrm{~Hz}$, which are 10,9, and 8, respectively. Scales with higher characteristic frequencies than the vibration frequencies are defined as smaller scales, whereas those with lower characteristic frequencies are defined as larger scales. Table 3 lists the definitions of the analyzed scalesbelow.

The turbulent intensity distribution is observed at various scales, which is represented by $u_{\mathrm{rms}}^{+}$. As shown in Fig. $6 \mathrm{a}-\mathrm{c}$, in the main region affected by the vibrations, i.e., $y^{+}=10-15$, the turbulent intensities are enhanced by the vibrations on smaller scales. Moreover, in case 2, 

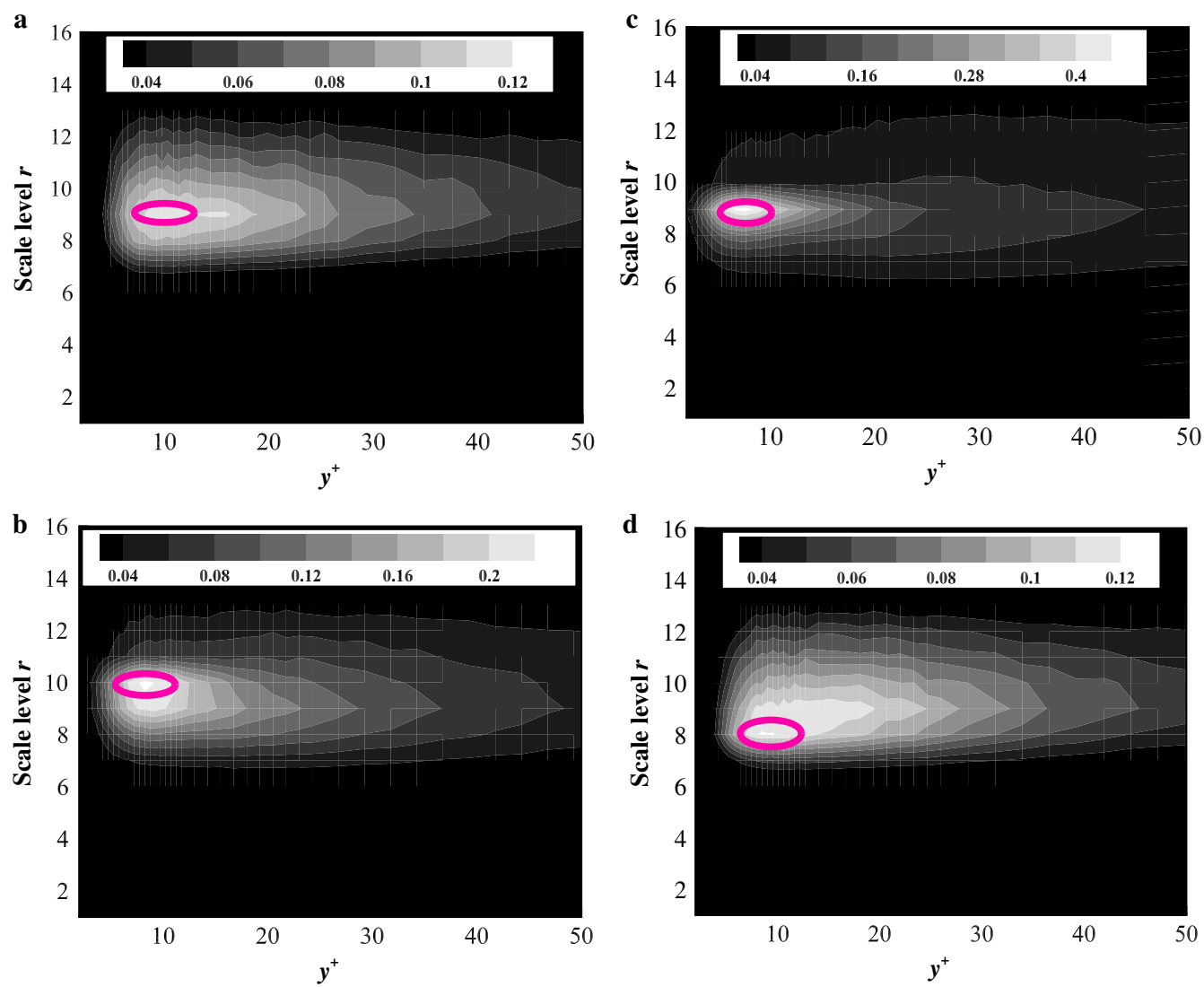

Fig. 4 Energy distribution with the scale and normal position under a the uncontrolled case, b $100 \mathrm{~V}$ and $80 \mathrm{~Hz}$, c $100 \mathrm{~V}$ and $160 \mathrm{~Hz}$, and d $100 \mathrm{~V}$ and $240 \mathrm{~Hz}$
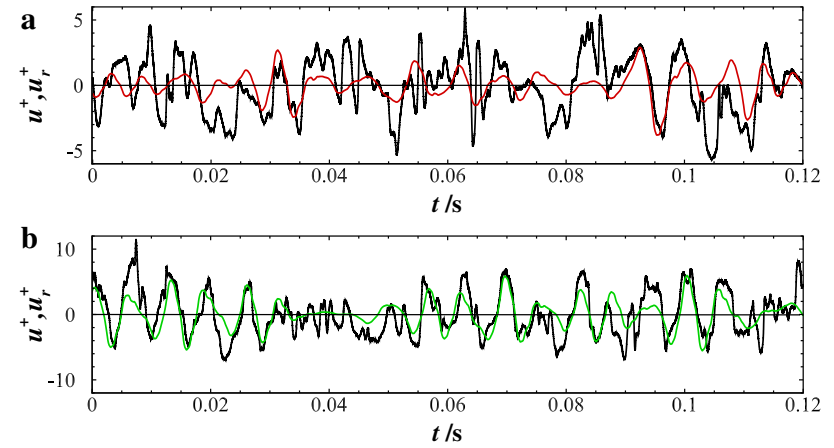

Fig. 5 Comparison between the streamwise fluctuating signal $u^{+}$ and reconstructed fluctuating signal $u_{r}^{+}$at a scale level of 9 for a the uncontrolled case, $u^{+}$: black line; $u_{r}^{+}:$red line, and $\mathbf{b}$ case $2, u^{+}$: black line; $u_{r}^{+}$: green line

the vibrations obviously weaken the intensities at larger scales; however, they have only a slight effect at larger scales in cases 1 and 3. Generally, in the near-wall region, the vibrations redistributed the energy to different scales [29].
Table 3 Definition of the analyzed scales

\begin{tabular}{llcll}
\hline Case & Parameters & $\begin{array}{l}\text { Vibration } \\
\text { scale }\end{array}$ & Smaller scale & Larger scale \\
\hline 1 & $100 \mathrm{~V}, 80 \mathrm{~Hz}$ & 10 & $<10$ & $>10$ \\
2 & $100 \mathrm{~V}, 160 \mathrm{~Hz}$ & 9 & $<9$ & $>9$ \\
3 & $100 \mathrm{~V}, 240 \mathrm{~Hz}$ & 8 & $<8$ & $>8$ \\
\hline
\end{tabular}

\subsection{Skewness}

The skewness, $\left\langle u_{r}^{\prime 3}\right\rangle /\left\langle u_{r}^{\prime 2}\right\rangle^{3 / 2}$, describes the symmetry of the turbulence fluctuations' probability distribution function (PDF). Positive skewness indicates that highmomentum sweep events with positive streamwise fluctuations are dominant, and negative skewness corresponds to low-speed eject events with negative streamwise fluctuations. Based on the wavelet decomposition, the wallnormal distributions of the skewness at different scales are shown in Fig. 7a-c. In case 2, the skewness value decreases dramatically, whereas it experiences almost no change in case 3 . The skewness is positive in the 

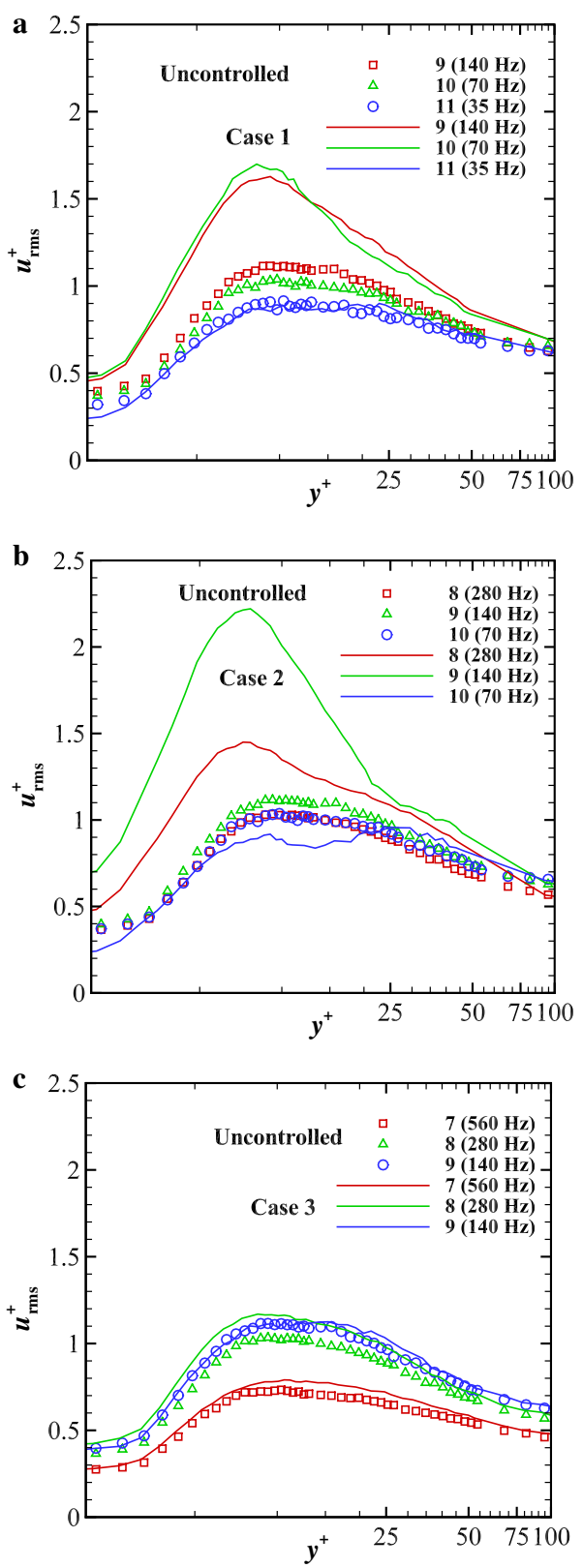

Fig. 6 Comparison of the turbulence intensities at different scales between the uncontrolled case and cases $\mathbf{a} 1, \mathbf{b} 2$, and $\mathbf{c} 3$

near-wall region of $y^{+}=10-15$ for all cases. For cases 1 and 2 , the skewness in the near-wall region decreases (close to zero) significantly, implying a better symmetry of the PDF for streamwise fluctuations. Then likely reason is that the PZT actuator induces flow structures with a certain frequency and highly fluctuating amplitude, and that the generated structures result in an alteration of the sweep events in the near-wall region.
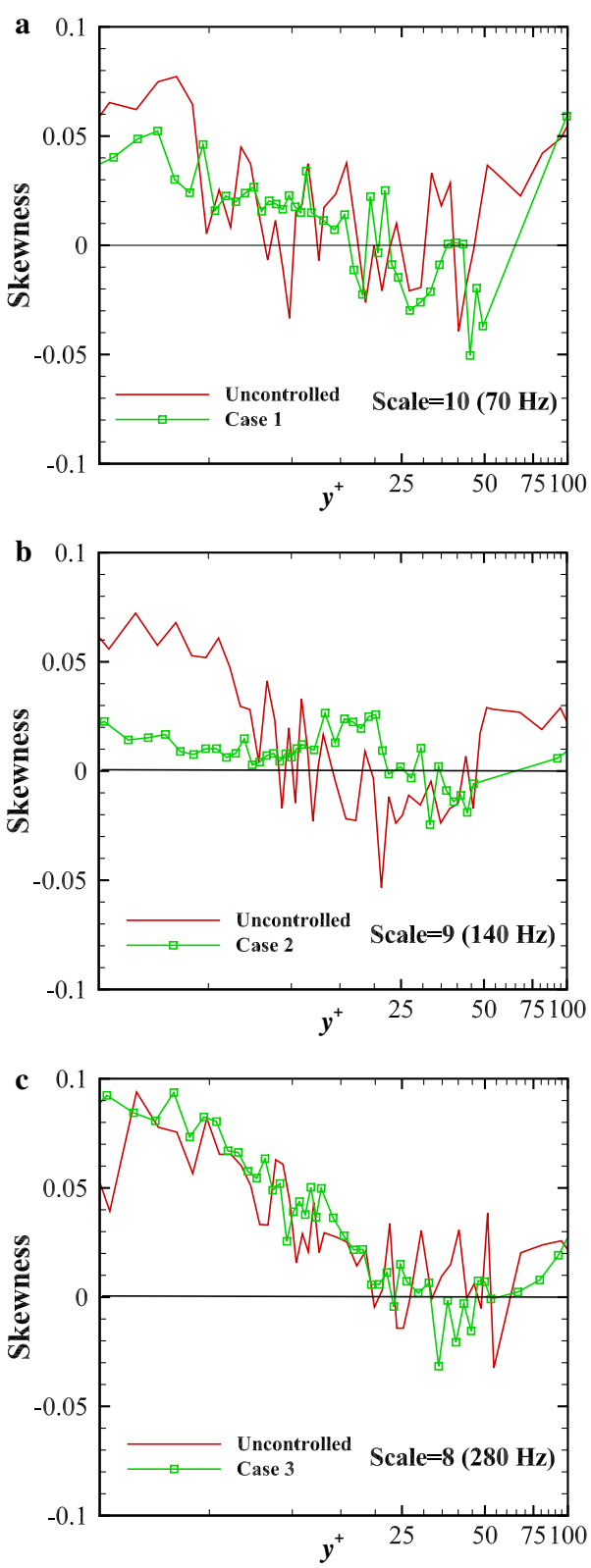

Fig. 7 Comparison of the skewness of the vibration scales between the uncontrolled case and cases $\mathbf{a} 1, \mathbf{b} 2$, and $\mathbf{c} 3$

\subsection{Flatness}

Figure 8 shows the distribution of the flatness factor, $\left\langle u_{r}^{\prime 4}\right\rangle /\left\langle u_{r}^{\prime 2}\right\rangle^{2}$, at different scales, which is a classic indicator of the turbulence fluctuation's intermittency. A flatness larger than 3 implies a strong intermittency introduced by sweep events with highly fluctuating momentum. As shown, the flatness of controlled cases decreases at smaller scales and vibration scales, and it has a very obvious drop over the 

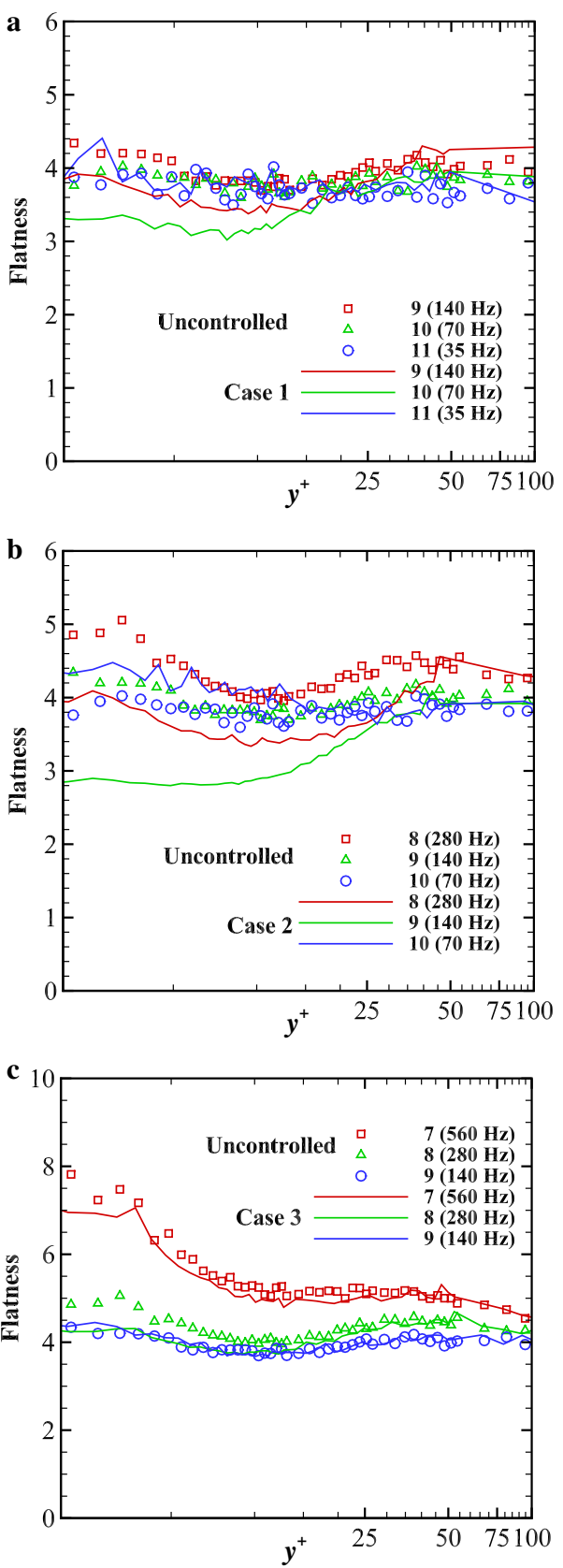

Fig. 8 Comparison of the flatness at different scales between the uncontrolled case and cases a 1, b 2, and $\mathbf{c} 3$

vibration scales. The variation in the flatness is positively correlated with the drag reduction effect. Therefore, this result indicates that the PZT actuator effectively reduces the intermittency and modulates the velocity fluctuation to be more periodic, especially for the vibration scales.

The PDFs of the streamwise fluctuation at $y^{+}=14$, where the inner spectral peak is located, are plotted in Fig. 9a-c according to the skewness and flatness results. The results of case 2 are presented and are compared with those of the uncontrolled case. The change in PDF
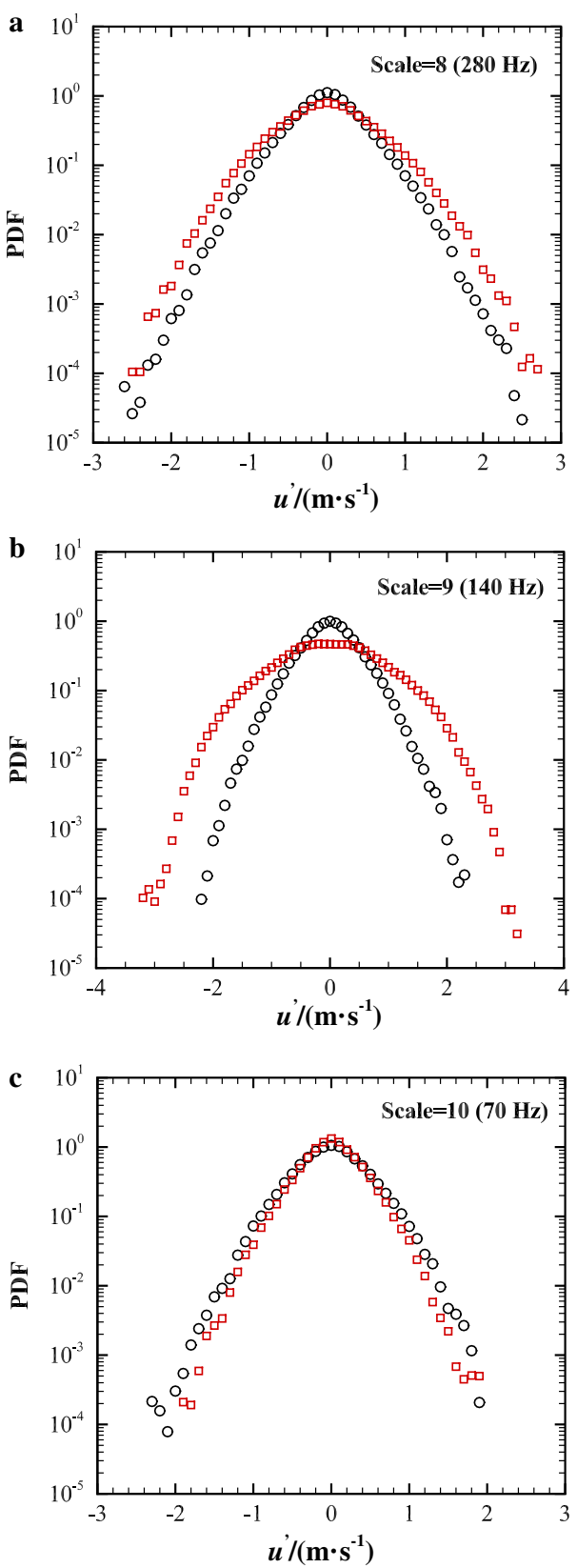

Fig. 9 Comparison of the velocity fluctuation PDFs at $y^{+}=14$ in the uncontrolled case (black circles) and case 2 (red squares) at a scale $=8 ; \mathbf{b}$ scale $=9 ;$ and $\mathbf{c}$ scale $=10$

is apparent under perturbation, as shown in Fig. 9a-c. At larger scales, the PZT control shortens the PDF tails, which means that the vibration concentrates the kinematic energy to the mean flow by weakening the largescale structures in the near-wall turbulence. These results agree with the previous conclusion. On the vibration and smaller scales, the vibration causes the PDF to have longer tails on both sides, implying an increase in the probability of high-amplitude fluctuations. It indicates that the fluctuations of the vibration and smaller scales have high 
intensity. Combined with the results of the flatness factor, it shows that the PZT disturbance caused fluctuations on the vibration and smaller scales with high intensity and low intermittency.

Based on the study reported by Jiang and Zhang [43], bursting process components are the fluctuations that cause the flatness factor (FF) to be larger than 3 . The two principal indicators used for our analysis of the data intermittency were the FF and local intermittency measure. FF is defined as follows:

$\mathrm{FF}(r)=\frac{u_{r}^{\prime 4}(t)_{t}}{u_{r}^{\prime 2}(t)_{t}^{2}}$

This function gives the level of intermittency at scale level $r$. Therefore, the difference with respect to the Gaussian statistics with an FF equal to 3 can be directly obtained.

The local intermittency measure (LIM) is defined as follows:

$\operatorname{LIM}(r, t)=\frac{u_{r}^{\prime 2}(t)}{u_{r}^{\prime 2}(t)_{t}}$

According to Camussi and Guj [40], LIM is a good qualitative indicator of the intermittency and magnitude of the energy fluctuations. Overall, detecting the bursting process indicates extracting the components in the fluctuations that make FF larger than 3 using these two indicators. The extraction procedure can be summarized as follows. For a certain scale, $L I M$ can be computed as a function of $t$ and threshold level $l$, can be set for LIM. The initial value of $l$ is set to $l_{0}=u_{\max }^{\prime 2} / u^{\prime 2}(t)_{t}$, where $u_{\max }^{\prime 2}$ is the maximum value of the fluctuations over the entire time domain at this scale. Then, FF of the fluctuation components $u^{\prime}\left(t_{i}\right)\left(\operatorname{LIM}\left(t_{i}\right)<l=l_{0}\right)$ can be calculated. If FF is larger than $3, l$ is reduced from $l_{0}$ by several times at a certain rate. At each time, the FF of the fluctuation components $u^{\prime}\left(t_{i}\right)\left(\operatorname{LIM}\left(t_{i}\right)<l\right)$ is calculated. The detecting process stops when the FF of $u^{\prime}\left(t_{i}\right)\left(\operatorname{LIM}\left(t_{i}\right)<l\right)$ drops to 3 and $l=l_{(\mathrm{FF}=3)}$. Finally, the fluctuation components $u^{\prime}\left(t_{j}\right)$ $\left(\operatorname{LIM}\left(t_{j}\right)>l_{(\mathrm{FF}=3)}\right)$ are extracted as the bursting process. The completion status of the detection process is shown in Fig. 10. The black line denotes the fluctuation at a scale level of 9 , whereas red line denotes the bursting processes detected at this scale.

According to this detection method, the statistical results of the bursting process can be studied at all scales. To obtain an overall understanding of the process, we superimposed the energy of the bursting process, $E_{\mathrm{b}}$, and total energy, $E$, at all scales. Their ratios are obtained in Fig. 11 a. $E_{\mathrm{b}} / E$, which represents the relative importance

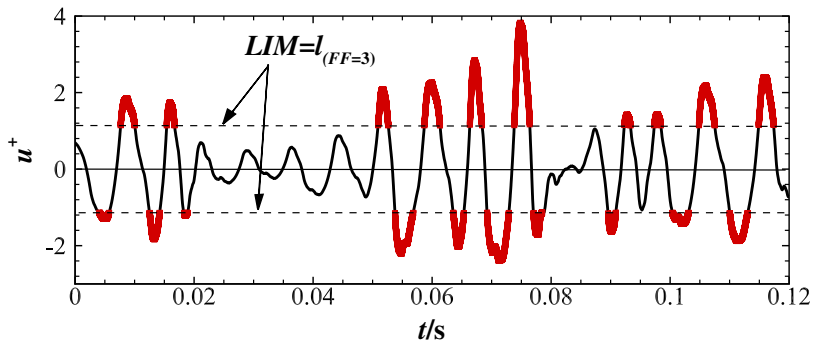

Fig. 10 Schematic diagram of the coherent structure extraction method
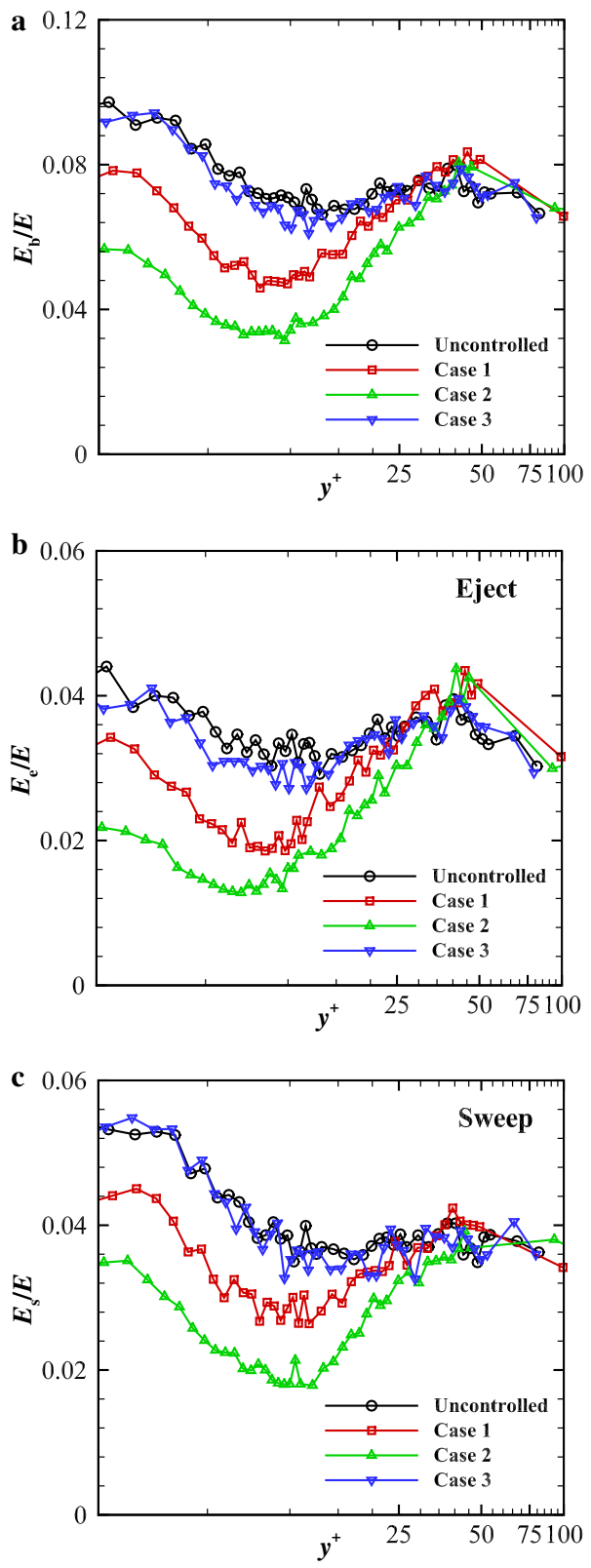

Fig. 11 Distribution of the energy ratio of a the bursting process $E_{\mathrm{b}}$ with respect to the original fluctuations $E$, b eject process $E_{\mathrm{e}}$ with respect to $E$, and $\mathbf{c}$ sweep process $E_{\mathrm{s}}$ with respect to $E$ 
of the bursting process, reflecting the fact that the vibration weakens the bursting process and that the case of $100 \mathrm{~V}$ and $160 \mathrm{~Hz}$ has the best performance. The impact is concentrated at $y^{+}<40$. These results are in agreement with those reported by Zheng et al. [18]. Combined with the drag reduction effect, it appears that the streamwise vortices in the buffer layer experience direct interference due to the PZT actuator, which leads to a breakdown in the burst mechanism in the inner region, thereby reducing the drag. The bursting process includes eject and sweep events with low- and high-speed fluids, respectively. To observe the impact of the vibration more carefully, the sweep and eject events were extracted according to the positive and negative fluctuation signals, respectively. Figure $11 \mathrm{~b}$, c show the proportion of eject and sweep event energy, i.e., $E_{\mathrm{e}}$ and $E_{\mathrm{s}}$, respectively, relative to $E$. As can be seen, the relative intensities of the eject and sweep events were both weakened by the vibration in the near-wall region. Even though the energy proportions of the eject and sweep events were different in the original fluctuations, the weakening degrees due to the vibrations were nearly same for the positive (sweep) and negative (eject) fluctuations. According to Fig. 7, the vibrations produced strong fluctuations in the positive and negative symmetries in the near-wall region; therefore, the impacts on the positive and negative signals were similar.

\section{Conclusions}

This experiment was conducted considering the Reynolds number of $R e_{\theta}=2183$ under three different conditions. The impact of a single PZT actuator on the statistical characteristics in the TBL was investigated using various methods. Statistics were gathered from the streamwise fluctuating velocity signals, measured using a single hot wire in the near-wall region. The major conclusions of the study are as follows.

1. From a multiscale point of view, in the near-wall region, the actuator redistributes the energy into different scales.

2. The changes in the skewness and flatness revealed that the PZT actuator, actuated at a certain frequency by a voltage with a sinusoidal waveform, effectively interferes with the sweep events, reduces the intermittency at smaller scales and on the vibration scales, and enhances the symmetry of the velocity signals at the vibration scales. Furthermore, the structure composition appears ordered. The PDF results indicate that the perturbation caused the fluctuations at the vibration scales and the smaller scales to have high intensity and low intermittency.

3. Based on the change in the flatness, the characteristics of the bursting events were analyzed. The results indi- cate that the vibrations restrain the bursting process for $y^{+}<40$, which indicates that the streamwise vortices in the buffer layer experience direct interference due to the PZT control, leading to a reduction in the drag.

Acknowledgements This work was supported by the National Natural Science Foundation of China (Grants 11732010, 11572221, 11872272, U1633109, 11802195) and the National Key R\&D Program of the Ministry of Science and Technology, China, on "Green Buildings and Building Industrialization" (Grant 2018YFC0705300).

Open Access This article is distributed under the terms of the Creative Commons Attribution 4.0 International License (http://creativeco mmons.org/licenses/by/4.0/), which permits unrestricted use, distribution, and reproduction in any medium, provided you give appropriate credit to the original author(s) and the source, provide a link to the Creative Commons license, and indicate if changes were made.

\section{References}

1. Xu, C.X., Deng, B.Q., Huang, W.X.: Coherent structures in wall turbulence and mechanism for drag reduction control. Sci. ChinaPhys. Mech. Astron. 56, 1053-1061 (2013)

2. Bai, H.L., Zhou, Y., Zhang, W.G., et al.: Active control of a turbulent boundary layer based on local surface perturbation. J. Fluid Mech. 750, 316-354 (2014)

3. Zheng, X.B., Jiang, N.: The spatial-temporal evolution of coherent structures in log law region of turbulent boundary layer. Acta. Mech. Sin. 31, 16-24 (2015)

4. Kim, J., Parviz, M., Robert, M.: Turbulence statistics in fully developed channel flow at low Reynolds number. J. Fluid Mech. 177, 133-166 (1987)

5. Blackwelder, R.F., Eckelmann, H.: Streamwise vortices associated with the bursting phenomenon. J. Fluid Mech. 94, 577-594 (1979)

6. Kim, J.: On the structure of wall-bounded turbulent flows. Phys. Fluids 26, 2088-2097 (1983)

7. Kline, S.J., Reynolds, W.C., Schraub, F.A., et al.: The structure of turbulent boundary layers. J. Fluid Mech. 30, 741-773 (1967)

8. Smith, C.R., Metzler, S.P.: The characteristics of low-speed streaks in the near-wall region of a turbulent boundary layer. J. Fluid Mech. 129, 27-54 (1983)

9. Walsh, M.J.: Riblets as a viscous drag reduction technique. AIAA J. 21, 485-486 (1983)

10. Bernard, P.S., Thomas, J.M., Handler, R.A.: Vortex dynamics and the production of Reynolds stress. J. Fluid Mech. 253, 385-419 (1993)

11. Wallace, J.M., Eckelmann, H., Brodkey, R.S.: The wall region in turbulent shear flow. J. Fluid Mech. 54, 39-48 (1972)

12. Haibao, H., Peng, D., Feng, Z., et al.: Effect of hydrophobicity on turbulent boundary layer under water. Exp. Therm. Fluid Sci. 60, $148-156$ (2015)

13. Tang, Z., Jiang, N., Zheng, X., et al.: Bursting process of large-and small-scale structures in turbulent boundary layer perturbed by a cylinder roughness element. Exp. Fluids 57, 79 (2016)

14. Choi, H., Moin, P., Kim, J.: Direct numerical simulation of turbulent flow over riblets. J. Fluid Mech. 255, 503-539 (1993)

15. Choi, H., Moin, P., Kim, J.: On the effect of riblets in fully developed laminar channel flows. Phys. Fluids 3, 1892-1896 (1991)

16. Kang, S., Choi, H.: Active wall motions for skin-friction drag reduction. Phys. Fluids 12, 3301-3304 (2000) 
17. Kim, J.: Control of turbulent boundary layers. Phys. Fluids 15, 1093-1105 (2003)

18. Rathnasingham, R., Breuer, K.S.: Active control of turbulent boundary layers. J. Fluid Mech. 495, 209-233 (2003)

19. Zheng, X., Jiang, N., Zhang, H.: Predetermined control of turbulent boundary layer with a piezoelectric oscillator. Chin. Phys. B 25, 014703 (2015)

20. Bird, J., Santer, M., Morrison, J.F.: Experimental control of turbulent boundary layers with in-plane travelling waves. Flow. Turbul. Combust. 100, 1015-1035 (2018)

21. Bai, J., Jiang, N., Zheng, X., et al.: Active control of wall-bounded turbulence for drag reduction with piezoelectric oscillators. Chin. Phys. B 27, 074701 (2018)

22. Caiazzo, A., Alujević, N., Pluymers, B., et al.: Active control of turbulent boundary layer-induced sound transmission through the cavity-backed double panels. J. Sound Vib. 422, 161-188 (2018)

23. McKeon, B.J., Jacobi, I., Duvvuri, S.: Dynamic roughness for manipulation and control of turbulent boundary layers: an overview. AIAA J. 56, 1-16 (2018)

24. Coller, B.D., Holmes, P., Lumley, J.L.: Control of bursting in boundary layer models. Appl. Mech. Rev. 47, S139-S143 (1994)

25. Jacobson, S.A., Reynolds, W.C.: Active control of streamwise vortices and streaks in boundary layers. J. Fluid Mech. 360, 179-211 (1998)

26. Han, Y., Zhang, H., Fan, B.C., et al.: Mechanism of controlling turbulent channel flow with the effect of spanwise Lorentz force distribution. Chin. Phys. B 26, 084704 (2017)

27. Choi, K.-S., Debisschop, J.-R., Clayton, B.R.: Turbulent boundary-layer control by means of spanwise-wall oscillation. AIAA J. 36, 1157-1163 (1998)

28. Zhao, P.L., Chen, Y.H., Dong, G., et al.: Experimental study on flow control of the turbulent boundary layer with micro-bubbles. Acta. Mech. Sin. 34, 830-838 (2018)

29. Tang, Z., Jiang, N., Zheng, X., et al.: Local dynamic perturbation effects on amplitude modulation in turbulent boundary layer flow based on triple decomposition. Phys. Fluids 31, 2 (2019)

30. Berger, T.W., Kim, J., Lee, C., et al.: Turbulent boundary layer control utilizing the Lorentz force. Phys. Fluids 12, 631-649 (2000)
31. Hutchins, N., Nickels, T.B., Marusic, I., et al.: Hot-wire spatial resolution issues in wall-bounded turbulence. J. Fluid Mech. 635, 103-136 (2009)

32. Ligrani, P.M., Bradshaw, P.: Spatial resolution and measurement of turbulence in the viscous sublayer using subminiature hot-wire probes. Exp. Fluids 5, 407-417 (1987)

33. Zheng, X.B., Jiang, N.: "Experimental study on spectrum and multi-scale nature of wall pressure and velocity in turbulent boundary layer. Chin. Phys. B 24, 6 (2015)

34. De Graaff, D.B., Eaton, J.K.: Reynolds-number scaling of the flat-plate turbulent boundary layer. J. Fluid Mech. 422, 319-346 (2000)

35. Stefes, B., Fernholz, H.H.: Skin friction and turbulence measurements in a boundary layer with zero-pressure-gradient under the influence of high intensity free-stream turbulence. Eur. J. Mech. B/Fluids 23, 303-318 (2004)

36. Patel, V.C., Head, M.R.: Some observations on skin friction and velocity profiles in fully developed pipe and channel flows. J. Fluid Mech. 38, 181-201 (1969)

37. Xin, Y.B., Xia, K.Q., Tong, P.: Measured velocity boundary layers in turbulent convection. Phys. Rev. Lett. 77, 1266-1269 (1996)

38. Tennekes, H., Lumley, J.L.: A first course in turbulence. MIT Press, Cambridge (1972)

39. Antoine, J.P.: Wavelet transforms and their applications. Phys. Today 56, 68 (2003)

40. Camussi, R., Guj, G.: Orthonormal wavelet decomposition of turbulent flows: intermittency and coherent structures. J. Fluid Mech. 348, 177-199 (1997)

41. Farge, M.: Wavelet transforms and their applications to turbulence. Ann. Rev. Fluid Mech. 24, 395-458 (1992)

42. Meneveau, C.: Analysis of turbulence in the orthonormal wavelet representation. J. Fluid Mech. 232, 469-520 (1991)

43. Nan, J., Jin, Z.: Detecting multi-scale coherent eddy structures and intermittency in turbulent boundary layer by wavelet analysis. Chin. Phys. Lett. 22, 1968-1971 (2005) 